\title{
Metabolomic and lipidomic approaches to identify biomarkers for bladder cancer and interstitial cystitis (Review)
}

\author{
MUHAMMAD SHAHID $^{1}$, AUSTIN YEON ${ }^{1}$ and JAYOUNG KIM ${ }^{1-5}$ \\ Departments of ${ }^{1}$ Surgery and ${ }^{2}$ Biomedical Sciences; ${ }^{3}$ Oschin Comprehensive Cancer Institute, Cedars-Sinai Medical Center, \\ Los Angeles, CA 90048; ${ }^{4}$ Department of Medicine, University of California, Los Angeles, CA 90024, USA; \\ ${ }^{5}$ Department of Urology, Ga Cheon University College of Medicine, Incheon 13120, Republic of Korea
}

Received May 21, 2020; Accepted September 18, 2020

DOI: $10.3892 / \mathrm{mmr} .2020 .11627$

\begin{abstract}
The discovery, introduction and clinical use of prognostic and diagnostic biomarkers has significantly improved outcomes for patients with various illnesses, including bladder cancer (BC) and other bladder-related diseases, such as benign bladder dysfunction and interstitial cystitis (IC). Several sensitive and noninvasive clinically relevant biomarkers for BC and IC have been identified. Metabolomic- and lipidomic-based biomarkers have notable clinical potential in improving treatment outcomes for patients with cancer; however, there are also some noted limitations. This review article provides a short and concise summary of the literature on metabolomic and lipidomic biomarkers for BC and IC, focusing on the possible clinical utility of profiling metabolic alterations in BC and IC.
\end{abstract}

\section{Contents}

1. Bladder cancer

2. Recently reported molecular subtype classification of BC

3. Interstitial cystitis

4. Metabolomics and lipidomics

5. Emerging technologies

Correspondence to: Professor Jayoung Kim, Department of Surgery, Cedars-Sinai Medical Center, Davis 5071, 8700 Beverly Blvd., Los Angeles, CA 90048, USA

E-mail: jayoung.kim@cshs.org

Abbreviations: $\mathrm{BC}$, bladder cancer; ESI, electrospray ionization; FGFR3, fibroblast growth factor receptor 3; GC, gas chromatography; IC, interstitial cystitis; LC, liquid chromatography; MAPP, Multidisciplinary Approach to the Study of Chronic Pelvic Pain; MIBC, muscle-invasive BC; MS, mass spectrometry; NMIBC, non-muscle-invasive $\mathrm{BC}$; NMR, nuclear magnetic resonance; TCA, tricarboxylic acid; TCGA, The Cancer Genome Atlas; UPLC, ultra-performance LC-MS

Key words: bladder cancer, interstitial cystitis, metabolomics, lipidomics, biomarkers
6. Applying metabolomics and lipidomics to $\mathrm{BC}$

7. Applying metabolomics and lipidomics to IC

8. Human specimens-based metabolomics and lipidomics biomarkers for BC

9. Human specimens-based metabolomics markers in IC 10. Conclusion

\section{Bladder cancer}

Bladder cancer (BC) is the most common malignancy of the urinary tract. In 2018, there were $\sim 549,000$ new cases of BC and 200,000 deaths related to BC globally (1). BC is the eighth most common cancer among men in the US (2) and is reported to affect men more frequently than women, with a ratio of 3.2:0.9 (1,2). In addition, the incidence of BC increases with age (3). According to the European Association of Urology, BC can be classified into two divergent phenotypes: Non-muscle-invasive BC (NMIBC) and muscle-invasive BC (MIBC). Furthermore, BC can be categorized into the following subtypes: Urothelial carcinoma, squamous epithelial carcinoma and adenocarcinoma (1-3). Urothelial carcinomas account for an overwhelming $90 \%$ of worldwide BC cases (4). Risk factors for BC include occupational factors, age, sex, race, socioeconomic status, personal health, diet and infection by pathogens (5-7). It is well-established that the progression of a normal cell to a cancer cell is a multistep process involving the accumulation of genetic alterations, referred to as carcinogenesis. NMIBC generally involves the mutation of fibroblast growth factor receptor 3 (FGFR3), giving rise to low-grade cancer that frequently recurs but seldom becomes invasive or progresses. By contrast, MIBC and carcinoma in situ exhibit deletions or mutations of TP53, RB transcriptional corepressor $1(R B 1)$, erb-b2 receptor tyrosine kinase 2 or $P T E N$, leading to high-grade and metastatic cancer (8). The emergence of high-throughput transcriptome sequencing techniques has assisted in the identification of versatile BC biomarkers, including long non-coding RNAs (lncRNAs), the aberrant expression of which can contribute to tumorigenesis in bladder tissues (9). lncRNA abhydrolase-domain containing 11 antisense RNA 1 (ABHD11-AS1) and lncRNA hypoxia-inducible factor $1 \alpha$ antisense RNA 2 (HIF1A-AS2) have been reported to be upregulated in BC tissues and cells, 
and their expression levels in tissues have been shown to be positively associated with advanced pathological grade and TNM classification $(10,11)$.

\section{Recently reported molecular subtype classification of $\mathrm{BC}$}

Classification systems for cancer are mainly based on pathological parameters, such as stage and grade. Such classification provides predictive prognostic information; however, for $\mathrm{BC}$, recurrence and progression vary widely from patient to patient, greatly affecting monitoring and treatment (12). The development of advanced techniques, such as sequencing and mass spectrometry (MS), and their implementation in omics, has provided better diagnostic and therapeutic information for the treatment of $\mathrm{BC}$ (13-16). Molecular subtyping, which is based on genetic characteristics, has made particularly notable progress in $\mathrm{BC}$ and is of increasing interest (17). The current molecular subtypes of BC share various characteristics, such as molecular features. However, these classifications can also vary, with two to seven distinct subtypes (18-20). As a result of this diversity, molecular classifications are not feasible for use in the clinical setting. This also highlights the need for a consensus on a single set of molecular subtypes that is applicable to clinical use. Nevertheless, the understanding of the biology of $\mathrm{BC}$ has been substantially improved by key achievements in molecular classification; for example, associations have been identified between molecular subtypes and urothelial differentiation, and similarities have been noted between $\mathrm{BC}$ subtypes and other types of cancer.

The first study into BC subtyping using molecular signatures was conducted at the University of Lund. This previous study examined the transcriptomes from 308 BC samples, identifying five different subtypes: Urobasal A, genomically unstable, urobasal $\mathrm{B}$, squamous cell carcinoma-like and infiltrated (2). In addition, two subtypes of high-grade MIBC were identified by examining published data from $262 \mathrm{BC}$ samples (18). The expression levels of keratin and $C D 44$ were analyzed, which were found to be related to differentiation of the urinary epithelium. Owing to similarities with expression profiles in breast cancer, these two molecular subtypes were named luminal-like and basal-like. Case studies of the luminal-like subtype revealed that it had much higher disease-specific and overall survival rates compared with the basal-like subtype, and the following transcription factors were enriched: FGFR 3 and tubercular sclerosis 1 . Specific changes were also noted in various pathways, including deletion mutations in the RB1 pathway and amplification of cyclin D1, E2F transcription factor 3 and cyclin E1 (18). In 2014, the MD Anderson Cancer Center analyzed mRNA expression patterns in 73 MIBC samples using molecular signatures identified from breast cancer studies. This led to the identification of three BC subtypes: Luminal, p53-like and basal (19). In addition to the aforementioned studies, The Cancer Genome Atlas (TCGA) has greatly improved biological databases and led to the updating of subtypes. Through genetic analysis of 129 patients with MIBC, TCGA identified four molecular BC subtypes: Clusters I, II, III and IV (20). This classification system was updated to include luminal, immune undifferentiated, luminal immune and basal subtypes based on genetic signatures, such as uroplakins and immune infiltration. Upon analysis of an additional 412 MIBC cases, TCGA classification of $\mathrm{BC}$ was further updated and consolidated into five subtypes: Luminal, luminal-infiltrated, basal-squamous, neural and luminal-papillary (21).

\section{Interstitial cystitis}

Interstitial cystitis (IC) is a chronic condition of unknown etiology with long-term notable pelvic/suprapubic pain and urinary storage symptoms, such as urgency, nocturia and frequency (22). The advent of cystoscopy led to major findings in IC, including bladder glomerulations during hydrodistention and Hunner's lesions (23). Although the epidemiology of IC is difficult to monitor due to its plethora of symptoms, recent studies have suggested an estimated prevalence of 100-300 per 100,000 women, and the prevalence rate is $\geq 10-20 \%$ lower in men $(24,25)$.

IC is generally diagnosed through exclusion; however, several attempts have been made to define standard diagnostic criteria. Recent guidelines set by the European Society for the Study of Interstitial Cystitis and the American Urological Association are currently being used worldwide to treat IC (26). Although treatment options for IC are limited and include hydrodistention, several oral pharmaceutical drugs have been approved by the US Food and Drug Administration, including pentosan polysulfate (elmiron), antihistamines, tricyclic antidepressants and immune modulators (27).

Owing to its unknown etiology, and large variability in sites of occurrence and symptom severity, IC is difficult to subtype. However, there is still an urgent need for a well-established and precise subtyping system. A recent study revealed that IC with Hunner's lesions displayed completely different histology, gene expression and prognoses compared to other forms of IC (28). IC can also be defined as a distinct non-inflammatory disorder characterized by preservation of the urothelium layer and symptom spread beyond the bladder without lesions (29).

\section{Metabolomics and lipidomics}

Metabolomics. Metabolomics is defined as the large-scale study of small molecules and metabolites involved in the regulation of metabolic pathways and their networks. Compared with genomics and proteomics, metabolomics is more closely linked to phenotypes; therefore, it can detect subtle changes in biological pathways under different physiological conditions and abnormal pathological processes. For the purposes of this review, we will focus on the application of metabolomics and lipidomics to $\mathrm{BC}$.

The aim of precision medicine is to create novel approaches to prevent disease and update clinical strategies to consider each individual's variability in terms of environment, lifestyle, genetics and molecular phenotypes (30). Metabolomics holds much promise for precision medicine and can be used to measure all metabolites in biological specimens (31). However, metabolomics presents significant analytical challenges over genomics and proteomics; it aims to measure molecules that range in polarity, from organic water-soluble acids to nonpolar lipids, which have disparate physical properties (32). As a complement to other omics techniques, metabolomics serves as a critical component of systems biology. Moreover, the study of 
metabolites and molecules is closely related to phenotypes and can improve understanding of intracellular metabolic alterations (31). The main aim of metabolomics is to identify altered metabolic pathways and biomarkers (33). Recent developments in metabolomics and statistical capabilities have improved the ability to investigate cancer metabolism and better understand cancer-related changes in metabolism, such as the conversion of glucose into the macromolecules needed for tumor cell proliferation and vascularization (34-36).

Lipidomics. Lipids are essential building blocks in the body that have several critical cellular functions and can provide information regarding ongoing lipid metabolism. The lipidome is the total lipid content in a cell (37). The emergence of lipidomics allows for the complete characterization of the cellular metabolome. Lipidomics may be the potential key to numerous metabolic diseases and can be utilized in several research areas, as well as in the development of diagnostic tools, drugs and therapeutic strategies (38). Lipidomics combined with bioinformatics can serve as a powerful tool for better understanding the biochemical mechanisms underlying lipid-related diseases by quantifying alterations in the levels of individual lipids, subclasses and molecular species, and identifying changes in pathways and networks (37). The emergence of metabolomics and lipidomics has enabled improved definition of differential metabolites in pathological conditions. Over the past two decades, metabolomics and lipidomics have seen significant advances, facilitated by rapid developments in novel analysis strategies, approaches, instruments and techniques (39).

\section{Emerging technologies}

Current development of methodologies. The physicochemical properties of all metabolites add additional complexity to metabolomics studies. To overcome these restrictions, various methods have been applied to overcome this complexity and challenges. MS and nuclear magnetic resonance (NMR) are the most frequently applied analytical approaches in metabolomics studies.

NMR spectroscopy. NMR is a nondestructive, nonbiased, easily quantifiable, fast and reproducible spectroscopy technique based on the principle that nuclei absorb and emit electromagnetic signals based on changes in the external magnetic field. NMR has several unique advantages in metabolomics (40). Metabolomics profiling by NMR is a powerful tool that can be used to diagnose a variety of diseases. NMR is based on the fact that nuclei, such as ${ }^{1} \mathrm{H},{ }^{13} \mathrm{C}$ and ${ }^{31} \mathrm{P}$, have nuclear spins and are able to exist at different energy levels in a magnetic field. Thus, these nuclei can generate valuable and identifiable information about metabolites. ${ }^{1} \mathrm{H}$ NMR is the most commonly used technique in metabolomics since ${ }^{1} \mathrm{H}$ is naturally abundant in biological samples. ${ }^{13} \mathrm{C}$ and ${ }^{31} \mathrm{P}$ NMR are used less frequently but can provide additional information on specific metabolites (40).

MS. MS-based metabolomics offers quantitative analysis of metabolites, ranging from measurement of a single molecule to thousands, with high selectivity and sensitivity. The combination of MS with separation techniques reduces the complexity of mass spectra by separating metabolites based on time, providing isobar separation and delivering additional information regarding physicochemical properties. To calculate the mass-to-charge ratio $(\mathrm{m} / \mathrm{z})$, MS acquires spectral data and relative intensity of the measured compounds. One potential drawback of MS-based techniques is the need for sample preparation, which can lead to potential loss of metabolites, changes in experimental conditions, discrimination of specific metabolite classes and other consequences $(41,42)$.

MS can effectively analyze small molecules separated by techniques, such as gas chromatography (GC), liquid chromatography (LC) and capillary electrophoresis. LC-MS and GC-MS can provide large amounts of chemical information for metabolomics studies. GC-MS uses the gaseous phase and achieves better metabolite separation than LC; however, unlike LC, GC typically requires chemical derivatization of the metabolic species prior to analysis. GC-MS is widely used in metabolomics studies as it can detect a wide range of intact metabolites with no need for chemical modification. For the separation of nonpolar to slightly polar molecules, traditional reverse-phase chromatography is used. Hydrophilic interaction LC is the technique of choice for separating strongly to slightly polar metabolites $(41,42)$.

Advantages and disadvantages. For metabolomics studies, each analytical technique has its own advantages and limitations. No single instrument or method can detect all metabolites accurately. Therefore, multiple methods and instruments are recommended to detect the greatest number of metabolites. For example, the Phenome Centre Birmingham utilizes LC-MS and NMR spectroscopy for metabolomic profiling and is able to detect a higher number of metabolites compared with using a single method alone (https:/www.birmingham.ac.uk/ research/activity/phenome-centre/about/index.aspx). Owing to the complexity of the metabolome, no single analytical method can fully discern the metabolome. NMR and MS each have their own strengths and weaknesses, which are described in previous publications (41-43).

\section{Applying metabolomics and lipidomics to $\mathrm{BC}$}

Applying metabolomics to $B C$. To diagnose initial or recurrent $\mathrm{BC}$, two standard diagnostic procedures are used: Cystoscopy and urine cytology. However, there are several limitations (43). As a result, there is an urgent need for a noninvasive, highly-sensitive, specific and convenient method for $\mathrm{BC}$ diagnosis. Urine is particularly suited for diagnostic purposes due to its availability, easy sample collection and storage in the malignant bladder $(42,44)$.

Both MS and NMR are used to analyze the metabolic profile and have become critical techniques for quantitatively and qualitatively measuring the metabolome. Both techniques allow for extensive and rapid analysis of small-molecule metabolites (45). Metabolomics can be useful in cancer research, demonstrating its potential for not only identifying candidate biomarkers but also elucidating the mechanisms underlying cancer pathogenesis. Metabolomics has already been applied to several cancer types with encouraging results, including breast, prostate, lung and liver cancer (46-50). Sahu et al (51) identified metabolic signatures, including those 


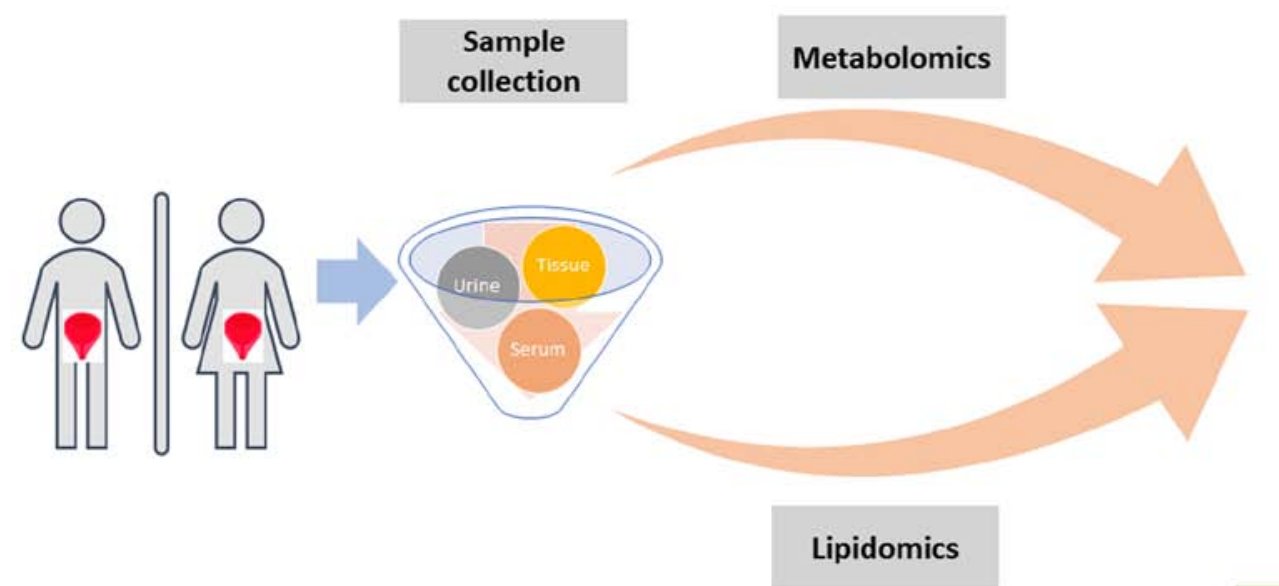

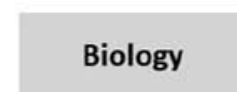

Robust

Biomarkers

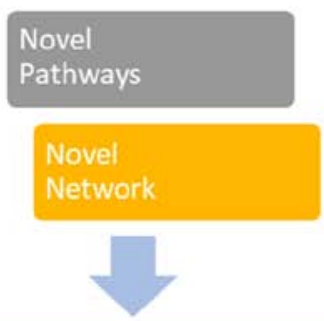

Novel Therapeutic targets, Strategies,

Understanding of Cancer

Figure 1. Biomarker discovery in the clinical setting. The field of metabolomics and lipidomics has advanced with respect to technological development. Large-scale datasets can help provide systems-scale information regarding diseases with inputs from metabolomics- and lipidomics-based analyses, which provide insightful biological data. This data can lead to robust and valid individual specific biomarkers for novel disease-specific pathways and networks. The application of new analytical technologies in omics studies should provide new information about promising drug therapeutics and improve understanding of the diseases.

for glucose, the tricarboxylic acid (TCA) cycle, lipids, amino acids and nucleotide pathways, by profiling the global metabolome using GC-MS and LC-MS. The results of this previous study revealed alterations in numerous pathways between normal urothelium and high-grade urothelial carcinoma at different stages. Recently, novel analytical methods have been developed using reverse-phase-high performance LC coupled with triple quadrupole MS for quantitatively determining and validating previously identified BC metabolites $(52,53)$. Clinical validation has previously been performed using urine samples from 40 patients with BC and matched controls, and suggested that the recovery and precision values were within the ranges set by FDA guidelines (52). Jin et al (53) performed LC-MS-based profiling of metabolites and identified distinctive metabolites in $138 \mathrm{BC}$ samples and 121 controls. This previous study identified 12 putative glycolysis- and $\beta$-oxidation-related markers. Multivariate regression analysis was then applied to confirm the association between the metabolic profiles and survival.

Applying lipidomics to $B C$. Current technologies allow for lipidomic analysis of a wide variety of biological specimens derived from animal models and clinical samples (54). The most appropriate analytical technique is selected based on the characteristics of the biological sample and the chemical properties of the targeted lipids. NMR and MS are accepted as the most powerful tools for phospholipid structure identification (55). Owing to structural diversity across phospholipid classes, analytical methods for lipidomics are continuously being improved. Notable progress has been made in lipid research by coupling MS with chromatographic separations. Soft ionization techniques, including matrix-assisted laser desorption/ionization and electrospray ionization (ESI), are good examples (56). Dill et al (57) used desorption ESI-imaging MS to investigate lipid species as diagnostic biomarkers of human BC compared to adjacent normal bladder tissue samples. The results revealed significant differences in the levels of glycerophosphoinositols, glycerophosphoserines, and fatty acids in tumor tissues compared with those in normal samples. Our group previously used ultra-performance LC-MS (UPLC-MS) to identify 1,864 differentially expressed lipids in cisplatin-resistant BC cells (58). Another of our lipidomics studies on cisplatin resistance of BCdemonstrated that acyl-CoA synthetase short chain family member 2 inhibition perturbed lipid metabolism, suggesting that cisplatin-resistant BC may have a specific lipidomic profile (58). Previously reported metabolomic biomarker candidates are summarized in Table I.

\section{Applying metabolomics and lipidomics to IC}

Applying metabolomics to IC. Chronic bladder pain is a hallmark of IC. Metabolomics studies can be used to analyze the characteristics of the disease state and identify novel approaches for reducing symptoms (41). Kind et al (59) performed global metabolomics profiling using various platforms, including NMR and LC-MS. Utilizing urine from patients with IC, this previous study profiled 490 metabolites, including histidine, erythronic acid and tartaric acid, and identified those with the highest fold changes. The identified metabolites were found to be associated with IC, suggesting its possible clinical use in urinary IC diagnosis. Using an MS-based metabolomics approach, the central clinical protocol of the Multidisciplinary Approach to the Study of Chronic Pelvic Pain (MAPP) Research Network, the Trans-MAPP Epidemiology and Phenotyping discovered urinary biomarkers in female patients with IC who underwent extensive urologic and non-urologic phenotyping (60). Parker et al (61) used LC-MS to identify molecular correlates of IC from urine obtained from female patients. This previous study identified a novel biomarker, etiocholan-3 $\alpha$-ol-17-one sulfate (Etio-S), a steroid metabolite, 
Table I. List of metabolomic biomarkers in BC.

\begin{tabular}{|c|c|c|c|c|c|c|c|c|}
\hline $\begin{array}{l}\text { First author, } \\
\text { year }\end{array}$ & Biomarker & Method & Sample size & $\begin{array}{c}\text { Sensitivity } \\
(\%)\end{array}$ & $\begin{array}{c}\text { Specificity } \\
(\%)\end{array}$ & AUC & Notes & (Refs.) \\
\hline $\begin{array}{l}\text { Pasikanti et al, } \\
2013\end{array}$ & $\begin{array}{l}2,5 \text {-furandicarboxylic } \\
\text { acid, ribitol and } \\
\text { ribonic acid }\end{array}$ & $\begin{array}{l}\text { GCxGC/ } \\
\text { TOFMS }\end{array}$ & $\begin{array}{l}38 \mathrm{BC}, \\
61 \text { Controls }\end{array}$ & 71 & 100 & - & Decreased & (70) \\
\hline $\begin{array}{l}\text { Wittmann et al, } \\
2014\end{array}$ & Taurine & MS & $\begin{array}{l}95 \mathrm{BC}, \\
345 \text { Controls }\end{array}$ & - & - & - & Increased & (68) \\
\hline $\begin{array}{l}\text { Srivastava et al, } \\
2010\end{array}$ & Taurine & $\begin{array}{l}\text { NMR } \\
\text { spectroscopy }\end{array}$ & $\begin{array}{l}33 \mathrm{BC}, \\
37 \text { Controls }\end{array}$ & - & - & - & Increased & $(65)$ \\
\hline Jin et al, 2014 & $\begin{array}{l}\text { Glycolysis and } \\
\text { acylcarnitines }\end{array}$ & LC-QTOFMS & $\begin{array}{l}138 \mathrm{BC}, \\
121 \text { Controls }\end{array}$ & $85-91.3$ & $85-92.5$ & 0.93 & Increased & (53) \\
\hline
\end{tabular}

BC, bladder cancer; AUC, area under the curve; GC, gas chromatography; MS, mass spectrometry; LC-MS, liquid chromatography-MS; NMR, nuclear magnetic resonance; GCxGC/TOFMS, two-dimensional GC time-of-flight MS; LC-QTOFMS, LC-quadrupole time-of-flight MS.

as being associated with a phenotypic subgroup of highly symptomatic IC. To the best of our knowledge, there are no reports in the literature involving the use of lipidomics to identify lipid compounds associated with IC.

\section{Human specimens-based metabolomics and lipidomics biomarkers for $\mathrm{BC}$}

Diagnosis of BC is dependent on several variables, including sensitivity and specificity of the methods, the invasiveness of the procedures and cost. Currently, cystoscopy and urine cytology are the most commonly used methods; however, both have several critical drawbacks, the most important being their limitations for detecting early BC (62). Overall survival in $\mathrm{BC}$ is highly dependent on early detection (62). The discovery of clinically relevant BC biomarkers will provide clinical value for prognostication, stratification, and identification of patients at higher risk for recurrence and progression. It is only through these outcomes that better management and treatment of patients with $\mathrm{BC}$ can be achieved (50). Zhang et al (63) compiled the results of previous metabolomics studies to discover BC biomarkers using urine, blood, tissue and cell lines. However, there is still a lack of consensus surrounding the pathophysiology of $\mathrm{BC}$. Thus, there is a great need for noninvasive markers to differentially diagnose BC (64).

\section{BC biomarkers}

Human urine. Numerous studies have reported that biomarkers can be identified using metabolomics, and recent studies have identified biomarkers that are capable of detecting early BC and predicting response to chemotherapy or relapse $(53,65-70)$. Some diagnostic biomarker studies have already compared the metabolic profiles of urine samples from patients with $\mathrm{BC}$ and healthy controls. Using ${ }^{1} \mathrm{H}$ NMR, Srivastava et al (65) revealed significant differences in the urine concentrations of hippurate, citrate and taurine in patients with BC compared with those in healthy controls. Jin et al (53) hypothesized that patients with $\mathrm{BC}$ could be distinguished from healthy controls based on metabolic profiles. This previous study revealed that the metabolic components of glycolysis and acylcarnitines were increased in MIBC compared with those in NMIBC. Citrate levels, a key metabolite of the TCA cycle, are altered in BC (66). Other urinary metabolites, including citrate, succinate and hippurate, have also been shown to be reduced in $\mathrm{BC}$ compared with those in healthy controls $(67,68)$. Shen et al $(69)$ also identified three upregulated and downregulated metabolites in BC: Nicotinuric acid, trehalose and AspAspGlyTrp were upregulated, whereas inosinic acid, ureidosuccinic acid and GlyCysAlaLys were downregulated. Several other studies have confirmed these results (70). The major findings from these studies are listed in Table I.

Tissue samples. Putluri et al (71) identified LC-MS-based metabolomic signatures using $\mathrm{BC}$ tissue samples, benign adjacent tissues and healthy controls. This previous study aimed to identify potential biomarker candidates and identify biology-related processes in BC carcinogenesis. A total of 50 metabolites with significant differences between $\mathrm{BC}$ and healthy controls were detected. Tripathi et al (72) performed high-resolution magic angle spinning NMR analysis of benign and $\mathrm{BC}$ tissues. The results revealed that $\mathrm{BC}$ exhibited more metabolic abnormalities compared with benign or healthy samples. These results identified 22 clearly differentiated metabolites. Using the same tissue samples, these results were cross-validated via targeted GC-MS analysis, demonstrating the potential of these biomarkers in clinically diagnosing BC. Yang et al (73) examined 48 BC tissue samples treated with gemcitabine as well as adjacent normal tissues from 12 of those patients. Based on UPLC-Q-Exactive-MS analysis, 34 significantly altered metabolites were found to be associated with BC.

Blood serum samples. Blood serum-based studies have established ways of distinguishing patients with $\mathrm{BC}$ from healthy controls. Cao et al (74) examined the serum profiles of patients with high- or low-grade $\mathrm{BC}$; in addition, patients with urinary calculi (hematuria) were included in the control group. 
Table II. List of metabolomic biomarkers in IC.

\begin{tabular}{|c|c|c|c|c|c|c|c|c|}
\hline $\begin{array}{l}\text { First author, } \\
\text { year }\end{array}$ & Biomarker & Method & Sample size & $\begin{array}{c}\text { Sensitivity } \\
(\%)\end{array}$ & $\begin{array}{l}\text { Specificity } \\
(\%)\end{array}$ & AUC & Notes & (Refs.) \\
\hline $\begin{array}{l}\text { Parker et al, } \\
2016\end{array}$ & $\begin{array}{l}\text { Etiocholan-3 } \alpha \text {-ol-1 } \\
\text { 7-one sulfate }\end{array}$ & MS & $\begin{array}{l}40 \mathrm{IC}, \\
40 \text { Controls }\end{array}$ & 87.4 & 0.92 & 0.92 & Increased & (61) \\
\hline $\begin{array}{l}\text { Kind et al, } \\
2016\end{array}$ & $\begin{array}{l}\text { Erythronic acid, } \\
\text { histidine and } \\
\text { tartaric acid }\end{array}$ & GC/MS & $\begin{array}{l}42 \mathrm{IC}, \\
21 \text { Controls }\end{array}$ & - & - & 0.9 & Increased & (59) \\
\hline $\begin{array}{l}\text { Wen et al, } \\
2015\end{array}$ & $\begin{array}{l}\text { tyramine and } \\
\text { 2-oxoglutarate }\end{array}$ & NMR & $\begin{array}{l}43 \mathrm{IC}, \\
21 \text { Controls }\end{array}$ & - & - & - & Increased & (76) \\
\hline $\begin{array}{l}\text { Shahid et al, } \\
2018\end{array}$ & Menthol & GC-TOF-MS & $\begin{array}{l}10 \mathrm{IC}, \\
10 \text { Controls }\end{array}$ & - & - & - & Decreased & (79) \\
\hline
\end{tabular}

IC, interstitial cystitis; AUC, area under the curve; GC, gas chromatography; MS, mass spectrometry; NMR, nuclear magnetic resonance; GC-TOFMS, GC time-of-flight MS.

Statistical analysis revealed that the serum profiles of patients with BC differed from those of healthy controls and those of patients with calculi. Serum metabolic profiles also allowed for classification of low- and high-grade BC. The levels of isoleucine/leucine, tyrosine, phenylalanine, choline, lactate, glycine and citrate were also shown to be significantly lower in patients with $\mathrm{BC}$ compared with those in healthy controls, whereas lipid and glucose levels were higher in patients with BC. Notably, additional comparisons of metabolite levels between patients with low- and high-grade $\mathrm{BC}$ revealed that the levels of tyrosine, phenylalanine, lactate and glycine were comparatively higher in low-grade patients, whereas glucose levels were lower. In addition, Bansal et al (75) used blood serum samples from patients with low-grade and high-grade $\mathrm{BC}$ and healthy controls. A total of six metabolites, dimethylamine, malonate, lactate, glutamine, histidine and valine, were significantly altered in BC samples compared with those in controls. Notably, external validation via a double-blind study consisting of 106 patients with suspected BC confirmed the utility of these metabolites for early diagnosis of $\mathrm{BC}$.

\section{Human specimens-based metabolomics markers in IC}

IC can present as a long continuum of mild to severe symptoms. In recent years, novel metabolomic techniques have been applied to gain a better understanding of disease mechanisms and uncover novel biomarkers (76). A previous study applied UPLC-MS-based metabolomics to examine urine samples from 10 patients with IC and 10 healthy controls. Phenylacetylglutamine was identified as a urinary marker of IC and was revealed to be elevated in the urine of patients with mild-to-moderate IC (77). In a separate study, Parker et al (61) used LC-MS to profile the metabolomes of urine samples from 40 patients with IC and matched controls. The results identified six metabolites as being closely associated with IC pathogenesis; one of which was Etio-S. Further analysis demonstrated that elevated Etio-S was a good predictor of IC, with sensitivity of $91.2 \%$, specificity of $87.4 \%$, and area under the curve of 0.92 . Longitudinal analysis of women in this cohort demonstrated that the differences in Etio-S persisted, indicating that these changes were long-lasting.

Taking an untargeted comprehensive metabolomic profiling approach, Kind et al (59) performed GC-MS analysis on urine specimens from patients with IC and healthy donors, and identified a total of 490 differentially expressed metabolites. Furthermore, Lamale et al (78) used urine samples from 40 women with IC and 29 healthy controls collected within a 24-h time frame. They discovered higher expression of three inflammatory markers, histamine, methylhistamine and IL-6, in patients with IC compared with those in the controls. In our previous biomarker discovery study, NMR-based global metabolomics analysis was applied to urine samples obtained from female patients with IC and matched healthy controls. The levels of tyramine and 2-oxoglutarate were significantly elevated in the IC urine specimens (76). Furthermore, in another of our previous studies, comprehensive solid-phase microextraction-GC-time-of-flight-MS profiling combined with bioinformatics analysis revealed that levels of volatile urinary metabolites, including menthol, were significantly reduced in patients with IC compared with those in normal controls (79). Previously reported metabolomics-based IC biomarker candidates are presented in Table II.

\section{Conclusion}

The present review aimed to understand the current development of biomarkers for bladder diseases based on various bioresources. In recent years, metabolomics and lipidomics has been widely used to understand the clinicopathology of the bladder and to discover the key differentially expressed metabolites or lipids specifically associated with bladder diseases. To determine the biological implications of metabolomic lipidomic signatures, bioinformatics tools, such as network and pathway enrichment analyses have been applied. The present review provided an overall summary of the metabolomics and lipidomic-based biomarker candidates for IC and BC (Fig. 1). 


\section{Acknowledgements}

Not applicable.

\section{Funding}

The authors acknowledge support from National Institutes of Health grants (grant nos. 1U01DK103260, 1R01DK100974, U24 DK097154 and NIH NCATS UCLA CTSI UL1TR000124), Department of Defense grants (grant nos. W81XWH-15-1-0415 and W81XWH-19-1-0109), Centers for Disease Controls and Prevention (grant no. 1U01DP006079), IMAGINE NO IC Research Grant, the Steven Spielberg Discovery Fund in Prostate Cancer Research Career Development Award, and the U.S.-Egypt Science and Technology Joint Fund. This research was partly supported by the Samuel Oschin Comprehensive Cancer Institute at Cedars-Sinai Medical Center through 2019 Lucy S. Gonda Award (to J.K.). In addition, this article is derived from the Subject Data funded in whole or part by National Academies of Sciences, Engineering, and Medicine (NAS) and The United States Agency for International Development (USAID). Any opinions, findings, conclusions, or recommendations expressed in this article are those of the authors alone, and do not necessarily reflect the views of USAID or NAS.

\section{Availability of data and materials}

Not applicable.

\section{Authors' contributions}

Research conception and design: JK. Data acquisition, data analysis and interpretation: MS and AY. Drafting of the manuscript: MS and AY. Critical revision of the manuscript: JK. Supervision: JK. All authors read and approved the final manuscript.

\section{Ethics approval and consent to participate}

Not applicable.

\section{Patient consent for publication}

Not applicable.

\section{Competing interests}

The authors declare they have no competing interests.

\section{References}

1. Bray F, Ferlay J, Soerjomataram I, Siegel RL, Torre LA and Jemal A: Global cancer statistics 2018: GLOBOCAN estimates of incidence and mortality worldwide for 36 cancers in 185 countries. CA Cancer J Clin 68: 394-424, 2018.

2. Sjodahl G, Lauss M, Lovgren K, Chebil G, Gudjonsson S, Veerla S, Patschan O, Aine M, Fernö M, Ringnér M, et al: A molecular taxonomy for urothelial carcinoma. Clin Cancer Res 18: 3377-3386, 2012.

3. Ferlay J, Soerjomataram I, Dikshit R, Eser S, Mathers C, Rebelo M, Parkin DM, Forman D and Bray F: Cancer incidence and mortality worldwide: Sources, methods and major patterns in GLOBOCAN 2012. Int J Cancer 136: E359-E386, 2015.
4. European Association of Urology (EAU): EAU Guidelines on Urological Infections. EAU Guidelines Office, Arnhem, 2018. https://uroweb.org/wp-content/uploads/EAU-Guidelines-onUrological-Infections-2018-large-text.pdf.

5. Czerniak B, Dinney C and McConkey D: Origins of bladder cancer. Annu Rev Pathol 11: 149-174, 2016.

6. Gruber K: Coffee consumption and bladder cancer are linked, analysis shows. BMJ 350: h1477, 2015.

7. Markowski MC, Boorjian SA, Burton JP, Hahn NM, Ingersoll MA, Maleki Vareki S, Pal SK and Sfanos KS: The microbiome and genitourinary cancer: A collaborative review. Eur Urol 75: 637-646, 2019.

8. Knowles MA and Hurst CD: Molecular biology of bladder cancer: New insights into pathogenesis and clinical diversity. Nat Rev Cancer 15: 25-41, 2015.

9. Zhang Q, Su M, Lu G and Wang J: The complexity of bladder cancer: Long noncoding RNAs are on the stage. Mol Cancer 12: 101, 2013.

10. Chen M, Li J, Zhuang C and Cai Z: Increased lncRNA ABHD11-AS1 represses the malignant phenotypes of bladder cancer. Oncotarget 8: 28176-28186, 2017.

11. Chen M, Zhuang C, Liu Y, Li J, Dai F, Xia M, Zhan Y, Lin J, Chen Z, He A, et al: Tetracycline-inducible shRNA targeting antisense long non-coding RNA HIF1A-AS2 represses the malignant phenotypes of bladder cancer. Cancer Lett 376: 155-164, 2016.

12. Lopez-Beltran A, Henriques V, Montironi R, Cimadamore A, Raspollini MR and Cheng L: Variants and new entities of bladder cancer. Histopathology 74: 77-96, 2019.

13. Boormans JL, Zwarthoff EC, Black PC, Goebell PJ, Kamat AM, Nawroth R, Seiler R, Williams SB and Schmitz-Dräger BJ: New horizons in bladder cancer research. Urol Oncol: March 7, 2019 (Epub ahead of print).

14. Kim S, Kim Y, Kong J, Kim E, Choi JH, Yuk HD, Lee H, Kim HR, Lee KH, Kang M, et al: Epigenetic regulation of mammalian Hedgehog signaling to the stroma determines the molecular subtype of bladder cancer. Elife 8: e43024, 2019.

15. Chen LM, Chang M, Dai Y, Chai KX, Dyrskjøt L, Sanchez-Carbayo M, Szarvas T, Zwarthoff EC, Lokeshwar V, Jeronimo C, et al: External validation of a multiplex urinary protein panel for the detection of bladder cancer in a multicenter cohort. Cancer Epidemiol Biomarkers Prev 23: 1804-1812, 2014.

16. Loras A, Suarez-Cabrera C, Martinez-Bisbal MC, Quintás G, Paramio JM, Martínez-Máñez R, Gil S and Ruiz-Cerdá JL: Integrative metabolomic and transcriptomic analysis for the study of bladder cancer. Cancers (Basel) 11: 686, 2019.

17. Sjodahl G, Jackson CL, Bartlett JM, Siemens DR and Berman DM: Molecular profiling in muscle-invasive bladder cancer: More than the sum of its parts. J Pathol 247: 563-573, 2019.

18. Damrauer JS, Hoadley KA, Chism DD, Fan C, Tiganelli CJ, Wobker SE, Yeh JJ, Milowsky MI, Iyer G, Parker JS and Kim WY: Intrinsic subtypes of high-grade bladder cancer reflect the hallmarks of breast cancer biology. Proc Natl Acad Sci USA 111: 3110-3115, 2014.

19. Choi W,Porten S,KimS, Willis D,Plimack ER,Hoffman-Censits J, Roth B, Cheng T, Tran M, Lee IL, et al: Identification of distinct basal and luminal subtypes of muscle-invasive bladder cancer with different sensitivities to frontline chemotherapy. Cancer Cell 25: 152-165, 2014.

20. Cancer Genome Atlas Research Network: Comprehensive molecular characterization of gastric adenocarcinoma. Nature 513: 202-209, 2014

21. Robertson AG, Kim J, Al-Ahmadie H, Bellmunt J, Guo G, Cherniack AD, Hinoue T, Laird PW, Hoadley KA, Akbani R, et al: Comprehensive molecular characterization of muscle-invasive bladder cancer. Cell 174: 1033, 2018.

22. Davis NF, Brady $\mathrm{CM}$ and Creagh $\mathrm{T}$ : Interstitial cystitis/painful bladder syndrome: Epidemiology, pathophysiology and evidence-based treatment options. Eur J Obstet Gynecol Reprod Biol 175: 30-37, 2014.

23. Fall M, Baranowski AP, Elneil S, Engeler D, Hughes J, Messelink EJ, Oberpenning F and de C Williams AC; European Association of Urology: EAU guidelines on chronic pelvic pain. Eur Urol 57: 35-48, 2010.

24. Hanno P, Lin A, Nordling J, van Ophoven A, Ueda T and Wein A; Bladder Pain Syndrome Committee of the International Consultation on Incontinence: Bladder pain syndrome committee of the international consultation on incontinence. Neurourol Urodyn 29: 191-198, 2010. 
25. Clemens JQ, Mullins C, Ackerman AL, Bavendam T, van Bokhoven A, Ellingson BM, Harte SE, Kutch JJ, Lai HH, Martucci KT, et al: Urologic chronic pelvic pain syndrome: Insights from the MAPP research network. Nat Rev Urol 16: 187-200, 2019.

26. van de Merwe JP, Nordling J, Bouchelouche P, Bouchelouche K, Cervigni M, Daha LK, Elneil S, Fall M, Hohlbrugger G, Irwin P, et al: Diagnostic criteria, classification, and nomenclature for painful bladder syndrome/interstitial cystitis: An ESSIC proposal. Eur Urol 53: 60-67, 2008.

27. Hanno PM, Burks DA, Clemens JQ, Dmochowski RR, Erickson D, Fitzgerald MP, Forrest JB, Gordon B, Gray M, Mayer RD, et al: AUA guideline for the diagnosis and treatment of interstitial cystitis/bladder pain syndrome. J Urol 185 2162-2170, 2011.

28. Akiyama Y, Maeda D, Katoh H, Morikawa T, Niimi A, Nomiya A Sato Y, Kawai T, Goto A, Fujimura T, et al: Molecular taxonomy of interstitial cystitis/bladder pain syndrome based on whole transcriptome profiling by next-generation RNA sequencing of bladder mucosal biopsies. J Urol 202: 290-300, 2019.

29. Peters KM, Killinger KA, Mounayer MH and Boura JA: Are ulcerative and nonulcerative interstitial cystitis/painful bladder syndrome 2 distinct diseases? A study of coexisting conditions. Urology 78: 301-308, 2011.

30. Seyhan AA and Carini C: Are innovation and new technologies in precision medicine paving a new era in patients centric care? J Transl Med 17: 114, 2019.

31. Clish CB: Metabolomics: An emerging but powerful tool for precision medicine. Cold Spring Harb Mol Case Stud 1: a000588, 2015.

32. Kuehnbaum NL and Britz-McKibbin P: New advances in separation science for metabolomics: Resolving chemical diversity in a post-genomic era. Chem Rev 113: 2437-2468, 2013.

33. Griffin JL and Shockcor JP: Metabolic profiles of cancer cells. Nat Rev Cancer 4: 551-561, 2004.

34. Vander Heiden MG: Targeting cancer metabolism: A therapeutic window opens. Nat Rev Drug Discov 10: 671-684, 2011

35. Pereira MM, Shori DK, Dormer RL and McPherson MA: Studies on phosphorylation of calcineurin. Biochem Soc Trans 18: 447, 1990.

36. Gatenby RA and Gillies RJ: Why do cancers have high aerobic glycolysis? Nat Rev Cancer 4: 891-899, 2004.

37. Han X: Lipidomics for studying metabolism. Nat Rev Endocrinol 12: 668-679, 2016.

38. Lydic TA and Goo YH: Lipidomics unveils the complexity of the lipidome in metabolic diseases. Clin Transl Med 7: 4, 2018.

39. Blanksby SJ and Mitchell TW: Advances in mass spectrometry for lipidomics. Annu Rev Anal Chem (Palo Alto Calif) 3 : 433-465, 2010

40. Emwas AH, Roy R, McKay RT, Tenori L, Saccenti E, Gowda GAN, Raftery D, Alahmari F, Jaremko L, Jaremko M and Wishart DS: NMR spectroscopy for metabolomics research. Metabolites 9: 123, 2019.

41. Fiehn $\mathrm{O}$ and Kim J: Metabolomics insights into pathophysiological mechanisms of interstitial cystitis. Int Neurourol J 18: 106-114, 2014

42. Chen $Z$ and Kim J: Urinary proteomics and metabolomics studies to monitor bladder health and urological diseases. BMC Urol 16: 11, 2016.

43. Zhu CZ, Ting HN, Ng KH and Ong TA: A review on the accuracy of bladder cancer detection methods. J Cancer 10: 4038-4044, 2019.

44. Kim J, Kim WT and Kim WJ: Advances in urinary biomarker discovery in urological research. Investig Clin Urol 61 (Suppl 1): S8-S22, 2020

45. Nicholson JK, Connelly J, Lindon JC and Holmes E: Metabonomics: A platform for studying drug toxicity and gene function. Nat Rev Drug Discov 1: 153-161, 2002.

46. Sreekumar A, Poisson LM, Rajendiran TM, Khan AP, Cao Q, Yu J, Laxman B, Mehra R, Lonigro RJ, Li Y, et al: Metabolomic profiles delineate potential role for sarcosine in prostate cancer progression. Nature 457: 910-914, 2009.

47. Jobard E, Pontoizeau C, Blaise BJ, Bachelot T, Elena-Herrmann B and Tredan O: A serum nuclear magnetic resonance-based metabolomic signature of advanced metastatic human breast cancer. Cancer Lett 343: 33-41, 2014.

48. Carrola J, Rocha CM, Barros AS, Gil AM, Goodfellow BJ, Carreira IM, Bernardo J, Gomes A, Sousa V, Carvalho L and Duarte IF: Metabolic signatures of lung cancer in biofluids: NMR-based metabonomics of urine. J Proteome Res 10: 221-230, 2011.
49. Chen F, Xue J, Zhou L, Wu S and Chen Z: Identification of serum biomarkers of hepatocarcinoma through liquid chromatography/mass spectrometry-based metabonomic method. Anal Bioanal Chem 401: 1899-1904, 2011.

50. Rodrigues D, Jeronimo C, Henrique R, Belo L, de Lourdes Bastos M, de Pinho PG and Carvalho M: Biomarkers in bladder cancer: A metabolomic approach using in vitro and ex vivo model systems. Int J Cancer 139: 256-268, 2016.

51. Sahu D, Lotan Y, Wittmann B, Neri B and Hansel DE: Metabolomics analysis reveals distinct profiles of nonmuscle-invasive and muscle-invasive bladder cancer. Cancer Med 6: 2106-2120, 2017.

52. Yumba Mpanga A, Siluk D, Jacyna J, Szerkus O, Wawrzyniak R, Markuszewski M, Matuszewski M, Kaliszan R and Markuszewski MJ: Targeted metabolomics in bladder cancer: From analytical methods development and validation towards application to clinical samples. Anal Chim Acta 1037: 188-199, 2018.

53. Jin X, Yun SJ, Jeong P, Kim IY, Kim WJ and Park S: Diagnosis of bladder cancer and prediction of survival by urinary metabolomics. Oncotarget 5: 1635-1645, 2014.

54. Yang K and Han X: Lipidomics: Techniques, applications, and outcomes related to biomedical sciences. Trends Biochem Sci 41: 954-969, 2016

55. Ellis DI, Dunn WB, Griffin JL, Allwood JW and Goodacre R: Metabolic fingerprinting as a diagnostic tool. Pharmacogenomics 8: 1243-1266, 2007.

56. Wang C, Wang M and Han X: Applications of mass spectrometry for cellular lipid analysis. Mol Biosyst 11: 698-713, 2015.

57. Dill AL, Eberlin LS, Costa AB, Zheng C, Ifa DR, Cheng L, Masterson TA, Koch MO, Vitek O and Cooks RG: Multivariate statistical identification of human bladder carcinomas using ambient ionization imaging mass spectrometry. Chemistry 17: 2897-2902, 2011

58. Lee MY, Yeon A, Shahid M, Cho E, Sairam V, Figlin R, Kim KH and Kim J: Reprogrammed lipid metabolism in bladder cancer with cisplatin resistance. Oncotarget 9: 13231-13243, 2018.

59. Kind T, Cho E, Park TD, Deng N, Liu Z, Lee T, Fiehn O and Kim J: Interstitial cystitis-associated urinary metabolites identified by mass-spectrometry based metabolomics analysis. Sci Rep 6: 39227, 2016.

60. Clemens JQ, Mullins C, Kusek JW, Kirkali Z, Mayer EA, Rodríguez LV, Klumpp DJ, Schaeffer AJ, Kreder KJ, Buchwald D, et al: The MAPP research network: A novel study of urologic chronic pelvic pain syndromes. BMC Urol 14: 57, 2014.

61. Parker KS, Crowley JR, Stephens-Shields AJ, van Bokhoven A, Lucia MS, Lai HH, Andriole GL, Hooton TM, Mullins C and Henderson JP: Urinary metabolomics identifies a molecular correlate of interstitial cystitis/bladder pain syndrome in a multidisciplinary approach to the study of chronic pelvic pain (MAPP) research network cohort. EBioMedicine 7: 167-174, 2016.

62. Mbeutcha A, Lucca I, Mathieu R, Lotan Y and Shariat SF: Current status of urinary biomarkers for detection and surveillance of bladder cancer. Urol Clin North Am 43: 47-62, 2016.

63. Zhang WT, Zhang ZW, Guo YD, Wang LS, Mao SY, Zhang JF, Liu MN and Yao XD: Discovering biomarkers in bladder cancer by metabolomics. Biomark Med 12: 1347-1359, 2018.

64. Kuo HC: Potential urine and serum biomarkers for patients with bladder pain syndrome/interstitial cystitis. Int J Urol 21 (Suppl 1): S34-S41, 2014

65. Srivastava S, Roy R, Singh S, Kumar P, Dalela D, Sankhwar SN Goel A and Sonkar AA: Taurine-a possible fingerprint biomarker in non-muscle invasive bladder cancer: A pilot study by $1 \mathrm{H}$ NMR spectroscopy. Cancer Biomark 6: 11-20, 2010.

66. Anderson NM, Mucka P, Kern JG and Feng H: The emerging role and targetability of the TCA cycle in cancer metabolism. Protein Cell 9: 216-237, 2018.

67. Pasikanti KK, Esuvaranathan K, Ho PC, Mahendran R, Kamaraj R, Wu QH, Chiong E and Chan EC: Noninvasive urinary metabonomic diagnosis of human bladder cancer. J Proteome Res 9: 2988-2995, 2010.

68. Wittmann BM, Stirdivant SM, Mitchell MW, Wulff JE, McDunn JE, Li Z, Dennis-Barrie A, Neri BP, Milburn MV, Lotan Y and Wolfert RL: Bladder cancer biomarker discovery using global metabolomic profiling of urine. PLoS One 9: e115870, 2014.

69. Shen C, Sun Z, Chen D, Su X, Jiang J, Li G, Lin B and Yan J: Developing urinary metabolomic signatures as early bladder cancer diagnostic markers. OMICS 19: 1-11, 2015. 
70. Pasikanti KK, Esuvaranathan K, Hong Y, Ho PC, Mahendran R, Raman Nee Mani L, Chiong E and Chan EC: Urinary metabotyping of bladder cancer using two-dimensional gas chromatography time-of-flight mass spectrometry. J Proteome Res 12: 3865-3873, 2013.

71. Putluri N, Shojaie A, Vasu VT, Vareed SK, Nalluri S, Putluri V, Thangjam GS, Panzitt K, Tallman CT, Butler C, et al: Metabolomic profiling reveals potential markers and bioprocesses altered in bladder cancer progression. Cancer Res 71: 7376-7386, 2011.

72. Tripathi P, Somashekar BS, Ponnusamy M, Gursky A, Dailey S, Kunju P, Lee CT, Chinnaiyan AM, Rajendiran TM and Ramamoorthy A: HR-MAS NMR tissue metabolomic signatures cross-validated by mass spectrometry distinguish bladder cancer from benign disease. J Proteome Res 12: 3519-3528, 2013.

73. Yang C, Sun X, Wang H, Lu T, Wu K, Guan Y, Tang J, Liang J, Sun R, Guo Z, et al: Metabolomic profiling identifies novel biomarkers and mechanisms in human bladder cancer treated with submucosal injection of gemcitabine. Int J Mol Med 44: 1952-1962, 2019

74. Cao M, Zhao L, Chen H, Xue W and Lin D: NMR-based metabolomic analysis of human bladder cancer. Anal Sci 28: 451-456, 2012.
75. Bansal N, Gupta A, Mitash N, Shakya PS, Mandhani A, Mahdi AA, Sankhwar SN and Mandal SK: Low- and high-grade bladder cancer determination via human serum-based metabolomics approach. J Proteome Res 12: 5839-5850, 2013.

76. Wen H,Lee T, You S, Park SH, Song H, Eilber KS, Anger JT, Freeman MR, Park S and Kim J: Urinary metabolite profiling combined with computational analysis predicts interstitial cystitis-associated candidate biomarkers. J Proteome Res 14: 541-548, 2015.

77. Fukui Y, Kato M, Inoue Y, Matsubara A and Itoh K: A metabonomic approach identifies human urinary phenylacetylglutamine as a novel marker of interstitial cystitis. J Chromatogr B Analyt Technol Biomed Life Sci 877: 3806-3812, 2009.

78. Lamale LM, Lutgendorf SK, Zimmerman MB and Kreder KJ: Interleukin-6, histamine, and methylhistamine as diagnostic markers for interstitial cystitis. Urology 68: 702-706, 2006.

79. Shahid M, Lee MY, Yeon A, Cho E, Sairam V, Valdiviez L, You S and Kim J: Menthol, a unique urinary volatile compound, is associated with chronic inflammation in interstitial cystitis. Sci Rep 8: 10859, 2018.

cc (i) $\Theta$ This work is licensed under a Creative Commons Attribution-NonCommercial-NoDerivatives 4.0 International (CC BY-NC-ND 4.0) License. 